OPEN ACCESS

Edited by:

Ming Jin Lim,

Guy's and St Thomas' NHS

Foundation Trust,

United Kingdom

Reviewed by:

Michael Eyre,

King's College London,

United Kingdom

Suvasini Sharma,

University of Delhi, India

*Correspondence:

Weihong Lin

linwh@jlu.edu.cn

Specialty section: This article was submitted to Multiple Sclerosis and Neuroimmunology, a section of the journal

Frontiers in Immunology

Received: 29 December 2021

Accepted: 11 February 2022

Published: 07 March 2022

Citation:

Zhong R, Chen Q, Zhang X, Zhang H and Lin W (2022) Risk Factors for

Mortality in Anti-NMDAR, Anti-LGI1, and Anti-GABABR Encephalitis.

Front. Immunol. 13:845365 doi: 10.3389/fimmu.2022.845365

\section{Risk Factors for Mortality in Anti-NMDAR, Anti-LGI1, and Anti-GABABR Encephalitis}

\author{
Rui Zhong ${ }^{1}$, Qingling Chen ${ }^{2}$, Xinyue Zhang ${ }^{1}$, Hanyu Zhang ${ }^{1}$ and Weihong Lin $^{1 *}$ \\ ${ }^{1}$ Department of Neurology, The First Hospital of Jilin University, Changchun, China, ${ }^{2}$ Department of Hepatology, Second \\ People's Clinical College of Tianjin Medical University, Tianjin, China
}

Objective: We aimed to investigate the mortality rate and identify the predictors of death in patients with anti-NMDAR, anti-LGl1, and anti-GABABR encephalitis.

Methods: Patients with anti-NMDAR, anti-LGl1, and anti-GABABR encephalitis were recruited from the Neurology Department of the First Hospital of Jilin University from March 2015 to November 2021. The primary outcome variable was a binary variable of death vs. survival. The potential risk factors for mortality were evaluated. The mortality rates were determined, and the independent predictors of death were identified using multivariable logistic regression analysis.

Results: A total of 100 hospitalized patients with anti-NMDAR, anti-LGl1, or anti-GABABR encephalitis were included in the final analysis. Fifteen patients (15\%) died during a median follow-up period of 18 months. The mortality rates were 10\% for anti-NMDAR encephalitis, 2.8\% for anti-LGl1 encephalitis, and $41.7 \%$ for anti-GABABR encephalitis. The multivariable analysis results showed that older age at onset [adjusted odds ratio $(O R)=1.017,95 \%$ confidence interval $(\mathrm{Cl})=1.009-1.136 ; p=0.023$ ] was independently associated with an increased risk of death. Antibody type was also associated with mortality. Patients with antiGABABR encephalitis had 13.458-fold greater odds of dying than patients with anti-LGl1 encephalitis (adjusted $\mathrm{OR}=13.458,95 \% \mathrm{Cl}=1.270-142.631 ; p=0.031$ ).

Conclusion: The general mortality rate of anti-NMDAR, anti-LGl1, and anti-GABABR encephalitis was $15 \%$. Age at onset and type of autoimmune encephalitis antibody were independent predictors of death in these patients.

Keywords: mortality, autoimmune encephalitis, anti-NMDAR encephalitis, anti-LGI1 encephalitis, antiGABABR encephalitis

\section{INTRODUCTION}

In recent years, the group of neuroinflammatory diseases known as autoimmune encephalitis (AE) has been recognized to encompass an increasingly wide and diverse range of pathological processes associated with the presence of antibodies against neuronal intracellular proteins, synaptic receptors, ion channels, and/or neuronal surface proteins (1-3). Many types of AE have been discovered in recent decades due to the development of biochemical assays; the most common types of neuronal surface antibody (NSAb)-associated AE include anti- $N$-methyl-D-aspartate receptor (NMDAR), anti- 
$\gamma$-aminobutyric acid $B$ receptor (GABABR), and anti-leucine-rich glioma-inactivated protein 1 (LGI1) encephalitis $(3,4)$. Immunotherapy improves the prognosis of most $\mathrm{AE}$ patients (5, 6) and is related to a decreased risk of clinical relapses of AE (7-9).

Mortality in AE patients has received little attention $(10,11)$. Hong and her colleagues reported high mortality in patients with anti-GABABR encephalitis; $32.1 \%$ of these patients died during the disease course (10). The mortality rate of anti-NMDAR encephalitis as reported by prior studies ranged from $5 \%$ to $11.46 \%$, which was lower than that of anti-GABABR encephalitis $(7,11,12)$. The predictors of death in $\mathrm{AE}$ patients also varied across prior studies $(10,11)$. The following clinical variables were identified as risk factors for death: number of complications, intensive care unit (ICU) admission, length of ICU stay, age at onset, presence of a tumor, and deep venous thrombosis $(10,11,13)$.

Data on the analysis of mortality in patients with antiNMDAR, anti-LGI1, and anti-GABABR encephalitis are lacking in Northeast China. Thus, we aimed to delineate the mortality rate in these patients and identify potential factors associated with death.

\section{METHODS}

\section{Study Design and Participants}

This retrospective observational study was conducted at a tertiary hospital serving a population of more than 5,000,000 per year in Northeast China. Hospitalized patients with antiNMDAR, anti-LGI1, and anti-GABABR encephalitis were recruited from the Neurology Department of the First Hospital of Jilin University from March 2015 to November 2021. The diagnosis of AE was confirmed in accordance with the diagnostic criteria established in 2016 (14). Cerebrospinal fluid (CSF) samples from all suspected $\mathrm{AE}$ patients were tested for $\mathrm{AE}$ antibodies using cell-based assays. These assays were performed with CSF rather than serum due to the superior quality of CSF samples. The other inclusion criteria were as follows: 1) the $\mathrm{AE}$ case must be newly diagnosed and 2) the patient must be in the acute phase of AE (defined as the first 3 months after the onset of AE symptoms). The exclusion criteria were as follows: 1) clinical and laboratory evidence of infectious encephalitis, caused by an agent such as Mycobacterium tuberculosis (TB) or a virus; 2) presence of other types of $\mathrm{AE}$ antibodies or neurological paraneoplastic antibodies; and 3) a follow-up period shorter than 6 months. The Ethics Committee of our hospital granted ethical approval for the retrospective observational study and waived the requirement for written informed consent. This study was performed in accordance with the Declaration of Helsinki.

\section{Data Collection}

For each eligible patient, the following demographic and clinical data were gathered retrospectively from our hospital electronic medical records and nursing records: gender, age at onset, clinical symptoms, presence of tumors, 24-h videoelectroencephalography (video-EEG) reporting, brain MRI findings, CSF analysis, antibody type, antibody titer, immunotherapy, and hospitalization data.

\section{Definition}

According to the International League Against Epilepsy (ILAE), tonic-clonic status epilepticus (SE) is defined as bilateral tonicclonic activity lasting longer than $5 \mathrm{~min}$, and absence SE and focal $\mathrm{SE}$ are defined by the occurrence of their respective clinical signs for more than $10 \mathrm{~min}$ (15). We adopted these definitions in the current study. Immunotherapy was considered to be delayed if the regimen was started at least 28 days after AE symptom onset (16, 17). We defined a long hospital stay as a hospital length of stay $>30$ days. Clinical relapse of AE was defined as new onset or worsening of symptoms occurring after an initial improvement or stabilization lasting at least 2 months (14). A CSF antibody titer $\leq 1: 10$ was scored as $(+)$, values $>1: 10$ but $\leq 1: 100$ as $(++)$, and values $>1: 100$ as $(+++)$. Hyperintensity on T2-weighted imaging (T2WI) and fluid-attenuated inversion recovery (FLAIR) imaging and hypointensity on T1-weighted imaging (T1WI) were defined as abnormal brain MRI. Abnormal EEG was defined as the occurrence of interictal epileptic discharges such as spikes or sharp waves. CSF analysis results were classified as abnormal or normal based on reference intervals.

\section{Outcome Assessment}

The primary outcome variable was a binary variable of death vs. survival. AE patients were followed up by clinic visits or telephone interviews for at least 6 months after discharge. The primary outcome assessments were performed at 1, 3, and 6 months after discharge and then every 6 months thereafter. Follow-up ended when the patient died during the disease course or was lost to follow-up.

\section{Statistical Analysis}

Continuous variables are presented as medians [interquartile ranges (IQRs)], and categorical variables are expressed as numbers and percentages. Continuous variables were compared using the Mann-Whitney $U$ tests between two groups or the Kruskal-Wallis tests between three groups, and Pearson's chisquared test or Fisher's exact test was used for categorical variables. Variables with $p<0.05$ in univariate analysis were retained in multivariable analysis. A multivariable logistic regression analysis was employed to identify the independent predictors of death, and the results are described as odds ratios (ORs) with 95\% confidence intervals (CIs). Subgroup analyses based on AE type were performed if there were more than three patients who died. All reported $p$-values were two-tailed and considered statistically significant at $<0.05$. All data were analyzed using IBM SPSS version 26.0 (SPSS, Chicago, IL, USA).

\section{RESULTS}

\section{Patient Characteristics}

In total, 100 hospitalized patients with anti-NMDAR, anti-LGI1, and anti-GABABR encephalitis were included in the final 
analysis after 10 patients were excluded based on the exclusion criteria. Among these patients, 40 had anti-NMDAR encephalitis, 36 had anti-LGI1 encephalitis, and 24 had antiGABABR encephalitis. The demographic and clinical characteristics of these patients are described in Table 1. In this cohort, which consisted of 54 men (54.0\%) and 46 women (46.0\%), the median age at onset was 52 years. Only one patient did not receive immunotherapy; however, immunotherapy was delayed in half of the patients. Forty-five (45\%) patients required admission to the ICU in the hospital. Eventually, 15 (15\%) patients died in the course of the disease. The median time interval from onset to death in the patients who died was 6 months, and the range was 1 to 18 months. The survival curve of the study participants is presented in Figure 1.

\section{Characteristics of Different Types of AE}

Table 1 also shows the patient characteristics by antibody type. Patients with anti-NMDAR encephalitis had the youngest age at onset ( 31 vs. 58 vs. 62 years, $p<0.001$ ). Patients with antiGABABR encephalitis had the highest prevalence of tumors; however, this difference was not statistically significant $(12.5 \%$ vs. $2.8 \%$ vs. $20.8 \%, p=0.084$ ). Patients with anti-LGI1 encephalitis were less likely to require admission to the ICU than those with anti-NMDAR or anti-GABABR encephalitis ( $60.0 \%$ vs. $13.9 \%$ vs. $66.7 \%$, respectively; $p<0.001)$. The case fatality rate also differed significantly among these three groups $(10.0 \%$ vs. $2.8 \%$ vs. $41.7 \%$, respectively; $p<0.001$ ).

\section{Univariate Analysis of Predictors of Death}

The median follow-up time was 18 months (IQR 10-30, range 684 months). During the follow-up period, 15 AE patients (15\%) died. Comparisons of variables between the death group and the survival group are shown in Table 2 . The age at onset in the death group was 63 years, which was significantly older than the onset age of 48 years in the survival group $(p=0.009)$. The occurrence of SE was more common in the death group than in the survival group $(66.7 \%$ vs. $37.6 \%, p=0.036)$. Patients with anti-GABABR encephalitis had an increased risk of death (66.7\% for antiGABABR vs. $16.5 \%$ overall for the two other types; $p<0.001$ ). ICU admission was significantly associated with an increased risk of mortality ( $73.3 \%$ vs. $40.0 \%, p=0.017)$. No other variable was found to be associated with mortality in this cohort of patients with anti-NMDAR, anti-LGI1, and anti-GABABR encephalitis.

\section{Multivariable Analysis of Predictors of Death}

A univariate analysis was performed to identify the variables associated with death in AE patients. Age at onset, occurrence of SE, antibody type, and ICU admission met the significance threshold of $p<0.05$ and were retained in the multivariable stepwise logistic regression models. The multivariable analysis results showed that older age at onset (adjusted OR $=1.017,95 \%$ $\mathrm{CI}=1.009-1.136 ; p=0.023$ ) was independently associated with an increased risk of death. Antibody type was also associated with death. The odds of dying were 13.458 times greater in patients with anti-GABABR encephalitis than in patients with anti-LGI1 encephalitis (adjusted OR $=13.458,95 \%$ CI $=1.270$ 142.631; $p=0.031$ ). The results are described in Table 3. No independent association was found between a history of SE or ICU admission and death $(p>0.05)$.

\section{Subgroup Analysis}

A subgroup analysis was performed based on AE antibody types. The risk factors for mortality in the subgroup with anti-LGI1

TABLE 1 | Patient characteristics by type of AE.

\begin{tabular}{|c|c|c|c|c|c|}
\hline Characteristics & Total $(n=100)$ & NMDAR $(n=40)$ & LGI1 $(n=36)$ & GABABR $(n=24)$ & $p$-value \\
\hline Men & $54(54.0)$ & $22(55.0)$ & $19(52.8)$ & $13(54.2)$ & 0.981 \\
\hline Age at onset (years) (median, IQR) & $52(33,63)$ & $31(21,42)$ & $58(49,66)$ & $62(56,65)$ & $<0.001$ \\
\hline Tumor presence & $11(11.0)$ & $5(12.5)$ & $1(2.8)$ & $5(20.8)$ & 0.084 \\
\hline EEG (abnormal) & $57(57.0)$ & $19(47.5)$ & $22(61.1)$ & $16(66.7)$ & 0.268 \\
\hline Brain MRI (abnormal) & $55(55.0)$ & $20(50.0)$ & $25(69.4)$ & $10(41.7)$ & 0.076 \\
\hline CSF analysis (abnormal) & $86(86.0)$ & $36(90.0)$ & $28(77.8)$ & $22(91.7)$ & 0.203 \\
\hline \multicolumn{6}{|l|}{ Antibody titer } \\
\hline+ & $31(31.0)$ & $17(42.5)$ & $10(27.8)$ & $4(16.7)$ & 0.26 \\
\hline++ & $51(51.0)$ & $17(42.5)$ & $20(55.6)$ & $14(58.3)$ & \\
\hline+++ & $18(18.0)$ & $6(15.0)$ & $6(16.7)$ & $6(25.0)$ & \\
\hline Administration of HGG & $85(85.0)$ & $37(92.5)$ & $24(66.7)$ & $15(62.5)$ & 0.006 \\
\hline Administration of corticosteroids & $76(76.0)$ & $35(87.5)$ & $31(86.1)$ & $19(79.2)$ & 0.647 \\
\hline Administration of plasma exchange & $5(5.0)$ & $4(10.0)$ & 0 & $1(4.2)$ & 0.133 \\
\hline Second-line immunotherapy & $6(6.0)$ & $4(10.0)$ & $2(5.6)$ & 0 & 0.262 \\
\hline Without immunotherapy & $1(1.0)$ & 0 & 0 & $1(4.2)$ & 0.202 \\
\hline Immunotherapy delay & $50(50.0)$ & $14(35.0)$ & $27(75.0)$ & $9(37.5)$ & 0.001 \\
\hline ICU admission & $45(45.0)$ & $24(60.0)$ & 5 (13.9) & $16(66.7)$ & $<0.001$ \\
\hline Relapse & $26(26.0)$ & $10(25.0)$ & $8(22.2)$ & 8 (33.3) & 0.619 \\
\hline Death & $15(15.0)$ & $4(10.0)$ & $1(2.8)$ & $10(41.7)$ & $<0.001$ \\
\hline Follow-up time (median, IQR) & $18(10,30)$ & $15(6,24)$ & $24(17,36)$ & $12(6,26)$ & 0.001 \\
\hline
\end{tabular}

$A E$, autoimmune encephalitis; IQR, interquartile range; NMDAR, N-methyl-D-aspartate receptor; LGI1, leucin-rich glioma inactivated-1; GABABR, g-aminobutyric acid type B receptor; CSF, cerebrospinal fluid; EEG, electroencephalogram; MRI, magnetic resonance imaging; HGG, human gamma globulin; ICU, intensive care unit.

+: antibody titer $\leq 1: 10$; ++: antibody titer $>1: 10$ and $\leq 1: 100$; +++: antibody titer $>1: 100$ as (+++). 


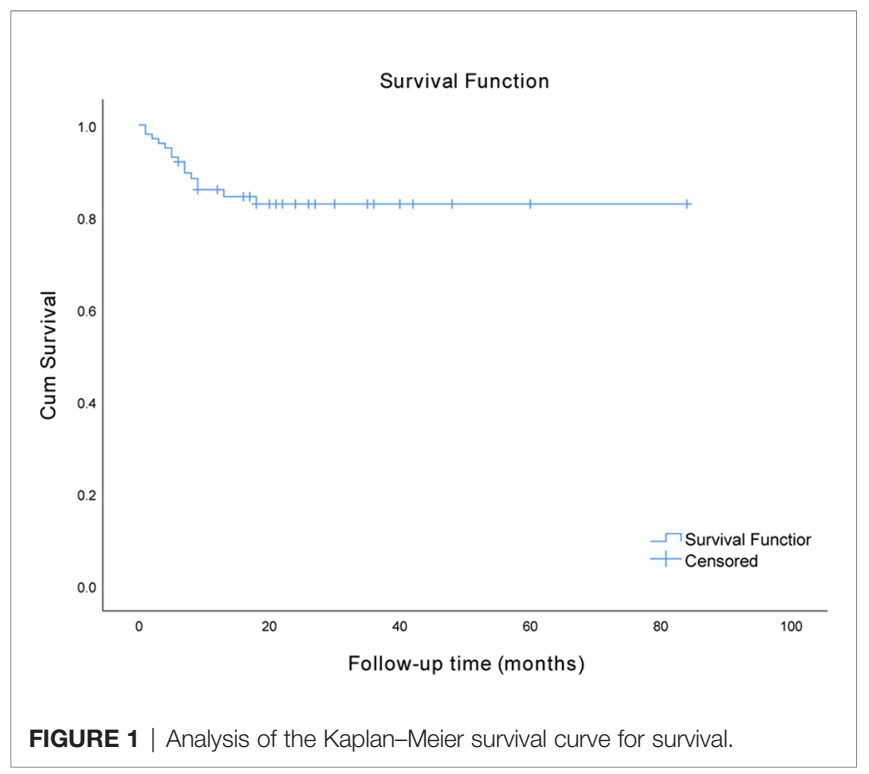

encephalitis were not investigated because only one patient died during the disease course. In anti-NMDAR encephalitis, no factor was found to be associated with the risk of death. As for patients with anti-GABABR encephalitis, a significant association was observed between older age at onset and a higher risk of mortality $(p=0.016)$. For details, see Table 4 .

\section{DISCUSSION}

In the present study, $15 \%$ of patients with anti-NMDAR, antiLGI1, or anti-GABABR encephalitis died during a median followup of 18 months. The mortality rates of anti-NMDAR encephalitis, anti-LGI1 encephalitis, and anti-GABABR encephalitis were $10 \%, 2.8 \%$, and $41.7 \%$, respectively. The study results suggested that older age at onset, $\mathrm{SE}$ occurrence, $\mathrm{AE}$ antibody type, and ICU admission were associated with the risk of death. Additionally, age at onset and AE antibody type were identified as independent predictors of death in these patients.

TABLE 2 | Univariate analysis of variables associated with death in the overall sample of patients.

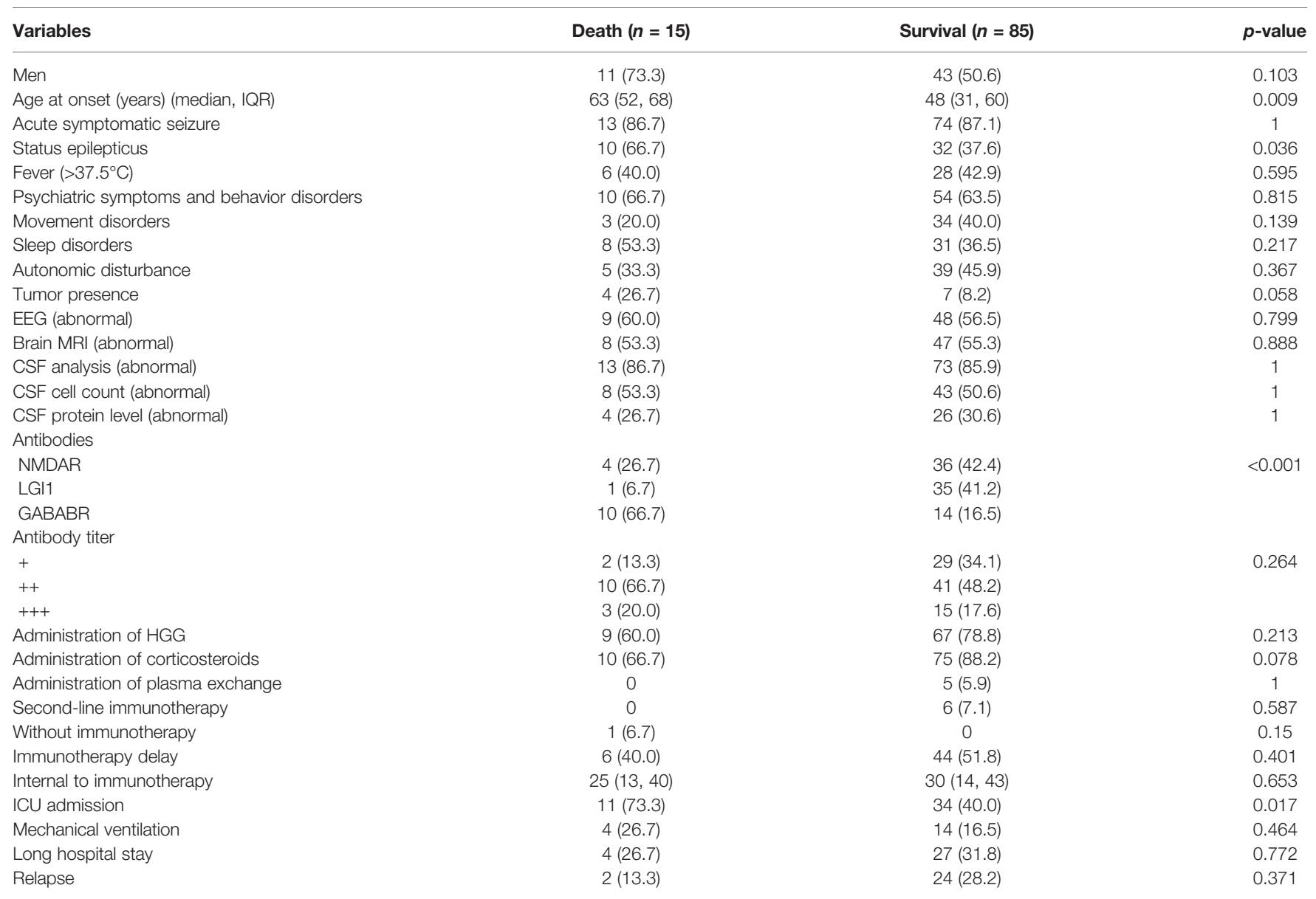

IQR, interquartile range; NMDAR, N-methyl-D-aspartate receptor; LGI1, leucin-rich glioma inactivated-1; GABABR, g-aminobutyric acid type B receptor; CSF, cerebrospinal fluid; EEG, electroencephalogram; MRI, magnetic resonance imaging; $H G G$, human gamma globulin; ICU, intensive care unit.

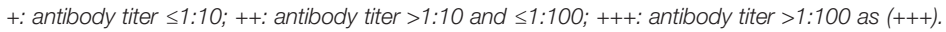


TABLE 3 | Multivariable analysis of variables independently associated with death in the overall sample of patients.

\begin{tabular}{|c|c|c|c|}
\hline Variables & Adjusted OR & $95 \% \mathrm{Cl}$ & $p$-value \\
\hline Age at onset & 1.071 & $1.009-1.136$ & 0.023 \\
\hline Status epilepticus & 1.928 & $0.445-8.355$ & 0.38 \\
\hline Antibodies type & & & 0.093 \\
\hline Antibodies type (1) & 11.974 & $0.806-177.892$ & 0.071 \\
\hline Antibodies type (2) & 13.458 & $1.270-142.631$ & 0.031 \\
\hline ICU admission & 2.281 & $0.478-10.876$ & 0.301 \\
\hline
\end{tabular}

ICU, intensive care unit; OR, odds ratio; $\mathrm{Cl}$, confidence interval.

Our study showed a general mortality rate of $15 \%$ in $\mathrm{AE}$ patients overall. In the subgroup with anti-NMDAR encephalitis, $10 \%$ of the patients died, which was in agreement with the $11.45 \%$ reported by Zhou et al. in West China (11). However, the mortality rate in our study was higher than the $5 \%$ reported in a cohort of patients with anti-NMDAR encephalitis in a multi-institutional observational study (7). This may be due to a treatment gap between developing countries and developed countries. Additionally, we found that the mortality rate was much higher in patients with antiGABABR encephalitis than in those with the other two antibody types. One explanation may be that there is a higher prevalence of underlying cancer in the anti-GABABR subgroup
$(13,18)$. It has been reported that the presence of cancer is associated with an increased risk of death in AE patients (10). In the present study, the mortality rate of anti-GABABR encephalitis (41.7\%) was similar to the rates reported by Maureille et al. in France (41.0\%) and by Lancaster et al. in the USA $(40.0 \%)(19,20)$. However, van Sonderen et al. reported a 2 -year case fatality rate of $19 \%$ in patients with anti-LGI1 encephalitis, which was higher than the rate in our cohort (21). The mortality rate of anti-LGI1 encephalitis may have been underestimated due to the relatively short follow-up period compared with the study by Titulaer et al. (21).

We found that older age at onset increased the risk of death in patients with anti-NMDAR, anti-LGI1, and anti-GABABR

TABLE 4 | Univariate analysis of variables associated with death in patients with anti-NMDAR and anti-GABABR encephalitis

\begin{tabular}{|c|c|c|c|c|c|c|}
\hline \multirow[t]{2}{*}{ Variables } & \multicolumn{2}{|c|}{ NMDAR $(n=40)$} & \multirow[t]{2}{*}{$p$-value } & \multicolumn{2}{|c|}{ GABABR $(n=24)$} & \multirow[t]{2}{*}{$p$-value } \\
\hline & Death $(n=4)$ & Survival $(n=36)$ & & Death $(n=10)$ & Survival $(n=14)$ & \\
\hline Men & $3(75.0)$ & $19(52.8)$ & 0.751 & $7(70.0)$ & $6(42.9)$ & 0.368 \\
\hline Age at onset (years) (median, IQR) & $45(24,67)$ & $30(20,40)$ & 0.176 & $64(62,68)$ & $57(53,61)$ & 0.016 \\
\hline Acute symptomatic seizure & $3(75.0)$ & 30 (83.3) & 0.552 & $9(90.0)$ & $13(92.9)$ & 1 \\
\hline Status epilepticus & $2(50.0)$ & $13(36.1)$ & 1 & 7 (70.0) & $9(64.3)$ & 1 \\
\hline Fever $\left(>37.5^{\circ} \mathrm{C}\right)$ & $3(75.0)$ & $18(50.0)$ & 0.673 & $2(20.0)$ & $6(42.9)$ & 0.464 \\
\hline Psychiatric symptoms and behavior disorders & $3(75.0)$ & $26(72.2)$ & 1 & 7 (70.0) & $9(64.3)$ & 1 \\
\hline Movement disorders & $1(25.0)$ & $22(61.1)$ & 0.249 & $2(20.0)$ & $2(14.3)$ & 1 \\
\hline Sleep disorders & $2(50.0)$ & $14(38.9)$ & 1 & $6(60.0)$ & $3(21.4)$ & 0.092 \\
\hline Autonomic disturbance & $2(50.0)$ & $20(55.6)$ & 1 & $3(30.0)$ & $7(50.0)$ & 0.421 \\
\hline Tumor presence & 0 & 5 (13.9) & 1 & $4(40.0)$ & $1(7.1)$ & 0.149 \\
\hline EEG (abnormal) & $2(50.0)$ & $17(47.2)$ & 1 & $6(60.0)$ & $10(71.4)$ & 0.884 \\
\hline Brain MRI (abnormal) & $2(50.0)$ & $18(50.0)$ & 1 & $5(50.0)$ & $5(35.7)$ & 0.78 \\
\hline CSF analysis (abnormal) & $4(100.0)$ & $32(88.9)$ & 1 & $8(80.0)$ & $14(100.0)$ & 0.163 \\
\hline CSF cell count (abnormal) & $2(50.0)$ & $28(77.8)$ & 0.543 & $6(60.0)$ & $8(57.1)$ & 1 \\
\hline CSF protein level (abnormal) & $2(50.0)$ & $13(36.1)$ & 1 & $2(20.0)$ & $5(35.7)$ & 0.704 \\
\hline \multicolumn{7}{|l|}{ Antibody titer } \\
\hline+ & $1(25.0)$ & $16(44.4)$ & 0.713 & $1(10.0)$ & $3(21.4)$ & 0.598 \\
\hline++ & $2(50.0)$ & $15(41.7)$ & & 7 (70.0) & 7 (50.0) & \\
\hline+++ & $1(25.0)$ & 5 (13.9) & & $2(20.0)$ & $4(28.6)$ & \\
\hline Administration of HGG & $3(75.0)$ & $34(94.4)$ & 0.277 & $6(60.0)$ & $9(64.3)$ & 1 \\
\hline Administration of corticosteroids & $2(50.0)$ & $33(91.7)$ & 0.069 & $7(70.0)$ & $12(85.7)$ & 0.671 \\
\hline Administration of plasma exchange & 0 & $4(11.1)$ & 1 & 0 & $1(7.1)$ & 1 \\
\hline Second-line immunotherapy & 0 & $4(11.1)$ & 1 & 0 & 0 & - \\
\hline Without immunotherapy & 0 & 0 & - & $1(10.0)$ & 0 & 0.417 \\
\hline Immunotherapy delay & $1(25.0)$ & $13(36.1)$ & 1 & $4(40.0)$ & $5(35.7)$ & 1 \\
\hline Internal to immunotherapy & $21(17,29)$ & $18(13,35)$ & 0.68 & $23(10,40)$ & $23(12,30)$ & 0.796 \\
\hline ICU admission & $2(50.0)$ & $22(61.1)$ & 1 & $8(80.0)$ & $8(57.1)$ & 0.464 \\
\hline Mechanical ventilation & $1(25.0)$ & 12 (33.3) & 1 & 3 (30.0) & $1(7.1)$ & 0.355 \\
\hline Long hospital stay & $2(50.0)$ & $22(61.1)$ & 1 & $2(20.0)$ & $1(7.1)$ & 0.754 \\
\hline Relapse & $1(25.0)$ & $9(25.0)$ & 1 & 1 (10.0) & 7 (50.0) & 0.107 \\
\hline
\end{tabular}

IQR, interquartile range; NMDAR, N-methyl-D-aspartate receptor; GABABR, g-aminobutyric acid type B receptor; CSF, cerebrospinal fluid; EEG, electroencephalogram; MRI, magnetic resonance imaging; HGG, human gamma globulin; ICU, intensive care unit.

+: antibody titer $\leq 1: 10$; ++: antibody titer $>1: 10$ and $\leq 1: 100$; +++: antibody titer $>1: 100$ as (+++). 
encephalitis. Our finding was supported by Hong and her colleagues (10). In their study, all patients who died were over 45 years old, and they proposed that patients aged 45 years or older had an increased risk of death (10). This may be because older AE patients were more likely to have underlying tumors and develop systemic complications that could lead to death (13). According to the literature, admission to the ICU was also more common in AE patients aged $\geq 18$ years than in those aged $<18$ years, indicating the relationship between age at onset and the severity of illness (22).

This study showed that AE patients in the death group were more likely to experience SE than those in the survival group. A recent study from West China also reported that refractory SE was one of the main causes of death in patients with antiNMDAR encephalitis (11). The occurrence of SE has been reported as one of the most common reasons for ICU admission $(13,23)$. A history of SE also predicts poor outcomes and death in patients with all-cause encephalitis (24). However, de Montmollin et al. noted that the occurrence of SE during the ICU stay was not a prognostic factor for poor neurological outcome in patients with anti-NMDAR encephalitis (25). In the present study, admission to the ICU was identified as another important predictor of death. Prior research also supported our finding (11). ICU management in AE patients frequently involves long-term care. A longer ICU stay increases the risk of complications such as pneumonia, deep venous thrombosis, and multiple organ dysfunction syndrome (MODS), which may threaten the patient's life $(13,18)$. A higher number of complications have been reported to be associated with a higher likelihood of death (10). Similarly, prior literature suggests that the lack of ICU admission was a prognostic factor for good outcomes in patients with antiNMDAR encephalitis $(7,9)$. However, a cohort from West China did not find a relationship between admission to the ICU and long-term functional outcomes in anti-NMDAR encephalitis (22). Thus, well-designed studies with large sample sizes are required to confirm our findings.

Our data showed that AE patients in the death group were more likely to have an underlying tumor than those in the survival group. However, this difference was not statistically significant $(p=0.058)$, which may be explained partly by the relatively small sample size. Another possible explanation could be that approximately $50 \%$ of patients with anti-GABABR encephalitis were reported previously to have an underlying tumor; however, only $11 \%$ of patients in our cohort had tumors (19). As for anti-NMDAR encephalitis, a similarly reduced tumor rate has also been observed in a Chinese cohort $(26,27)$. It is well known that patients can benefit greatly from awareness and knowledge of $\operatorname{AE}(26,28)$. However, there exists a knowledge gap regarding AE between China and developed countries. Thus, the differences may be a result of inadequate regular tumor screening and relatively short follow-up for some patients due to limited knowledge. We also observed a relatively low percentage of female patients in the anti-NMDAR encephalitis group, which may be explained partly by the reduced prevalence of ovarian teratoma in our cohort compared with Western cohorts. Tumor progression could contribute to an increased risk of death in anti-GABABR encephalitis $(19,29)$. Interestingly, the literature suggests the exciting possibility that the presence of tumors does not lead to a poor clinical outcome in patients with anti-NMDAR encephalitis $(9,22)$.

The present study has several limitations. First, the sample size in this cohort was relatively small, which may have led to limited statistical power. Relatedly, this small sample was drawn from a local tertiary hospital in Northeast China, and a regional selection bias may therefore exist. Second, the follow-up period was relatively short in some patients, which may cause the mortality rate to be underestimated in $\mathrm{AE}$ patients. These patients are still at risk of death over a longer period of time. Third, some patients with other types of antibodies, such as antiCaspr2 antibodies, were excluded, which may limit the interpretation of the analysis results for the types of $\mathrm{AE}$. Fourth, we did not assess the effect of tumor removal on the risk of death because data on the management of tumors were not available. Finally, the statistical significance threshold was not corrected for multiple comparisons, and variable selection for multivariable modeling according to univariate significance can be misleading $(30,31)$.

In summary, our data showed an overall mortality rate of $15 \%$ in patients with anti-NMDAR, anti-LGI1, or anti-GABABR encephalitis. Age at onset and type of AE antibody were identified as independent predictors of death in these patients.

\section{DATA AVAILABILITY STATEMENT}

The raw data supporting the conclusions of this article will be made available by the authors, without undue reservation.

\section{ETHICS STATEMENT}

The studies involving human participants were reviewed and approved by the medical ethical committees of the First Hospital of Jilin University. Written informed consent from the participants' legal guardian/next of kin was not required to participate in this study in accordance with the national legislation and the institutional requirements.

\section{AUTHOR CONTRIBUTIONS}

WL and RZ conceived and designed the study. RZ, HZ and XZ were involved in data acquisition. RZ and QC analyzed the data and wrote the manuscript. All authors contributed to the article and approved the submitted version.

\section{ACKNOWLEDGMENTS}

The authors would like to thank all the participants for their valuable data, cooperation, and participation. 


\section{REFERENCES}

1. Yeshokumar AK, Pardo CA. Autoimmune Epilepsies. Semin Pediatr Neurol (2017) 24:161-7. doi: 10.1016/j.spen.2017.08.012

2. Esposito S, Principi N, Calabresi P, Rigante D. An Evolving Redefinition of Autoimmune Encephalitis. Autoimmun Rev (2019) 18:155-63. doi: 10.1016/ j.autrev.2018.08.009

3. Wang Y, Yu Y, Hu Y, Li Y, Song F, Wang Y. Clinical and Electroencephalographic Features of the Seizures in Neuronal Surface Antibody-Associated Autoimmune Encephalitis. Front Neurol (2020) 11:280. doi: 10.3389/ fneur.2020.00280

4. Huang Q, Ma M, Wei X, Liao Y, Qi H, Wu Y, et al. Characteristics of Seizure and Antiepileptic Drug Utilization in Outpatients With Autoimmune Encephalitis. Front Neurol (2018) 9:1136. doi: 10.3389/fneur.2018.01136

5. Nosadini M, Mohammad SS, Ramanathan S, Brilot F, Dale RC. Immune Therapy in Autoimmune Encephalitis: A Systematic Review. Expert Rev Neurother (2015) 15:1391-419. doi: 10.1586/14737175.2015.1115720

6. Bien CG. Management of Autoimmune Encephalitis. Curr Opin Neurol (2021) 34:166-71. doi: 10.1097/WCO.0000000000000909

7. Titulaer MJ, McCracken L, Gabilondo I, Armangue T, Glaser C, Iizuka T, et al. Treatment and Prognostic Factors for Long-Term Outcome in Patients With Anti-NMDA Receptor Encephalitis: An Observational Cohort Study. Lancet Neurol (2013) 12:157-65. doi: 10.1016/S1474-4422(12)70310-1

8. Gabilondo I, Saiz A, Galan L, Gonzalez V, Jadraque R, Sabater L, et al. Analysis of Relapses in Anti-NMDAR Encephalitis. Neurology (2011) 77:9969. doi: 10.1212/WNL.0b013e31822cfc6b

9. Nosadini M, Eyre M, Molteni E, Thomas T, Irani SR, Dalmau J, et al. Use and Safety of Immunotherapeutic Management of N-Methyl-D-Aspartate Receptor Antibody Encephalitis: A Meta-Analysis. JAMA Neurol (2021) 78:1333-44. doi: 10.1001/jamaneurol.2021.3188

10. Lin J, Li C, Li A, Liu X, Wang R, Chen C, et al. Encephalitis With Antibodies Against the GABAB Receptor: High Mortality and Risk Factors. Front Neurol (2019) 10:1030. doi: 10.3389/fneur.2019.01030

11. Chi X, Wang W, Huang C, Wu M, Zhang L, Li J, et al. Risk Factors for Mortality in Patients With Anti-NMDA Receptor Encephalitis. Acta Neurol Scand (2017) 136:298-304. doi: 10.1111/ane.12723

12. Titulaer MJ, McCracken L, Gabilondo I, Iizuka T, Kawachi I, Bataller L, et al. Late-Onset Anti-NMDA Receptor Encephalitis. Neurology (2013) 81:105863. doi: 10.1212/WNL.0b013e3182a4a49c

13. Harutyunyan G, Hauer L, Dunser MW, Karamyan A, Moser T, Pikija S, et al. Autoimmune Encephalitis at the Neurological Intensive Care Unit: Etiologies, Reasons for Admission and Survival. Neurocrit Care (2017) 27:82-9. doi: 10.1007/s12028-016-0370-7

14. Graus F, Titulaer MJ, Balu R, Benseler S, Bien CG, Cellucci T, et al. A Clinical Approach to Diagnosis of Autoimmune Encephalitis. Lancet Neurol (2016) 15:391-404. doi: 10.1016/S1474-4422(15)00401-9

15. Trinka E, Cock H, Hesdorffer D, Rossetti AO, Scheffer IE, Shinnar S, et al. A Definition and Classification of Status Epilepticus-Report of the ILAE Task Force on Classification of Status Epilepticus. Epilepsia (2015) 56:1515-23. doi: 10.1111/epi.13121

16. Shen CH, Fang GL, Yang F, Cai MT, Zheng Y, Fang W, et al. Seizures and Risk of Epilepsy in Anti-NMDAR, Anti-LGI1, and Anti-GABAB R Encephalitis. Ann Clin Transl Neurol (2020) 7:1392-9. doi: 10.1002/acn3.51137

17. de Bruijn M, van Sonderen A, van Coevorden-Hameete MH, Bastiaansen A, Schreurs M, Rouhl R, et al. Evaluation of Seizure Treatment in Anti-LGI1, Anti-NMDAR, and Anti-GABABR Encephalitis. Neurology (2019) 92:e218596. doi: 10.1212/WNL.0000000000007475

18. Schubert J, Bramer D, Huttner HB, Gerner ST, Fuhrer H, Melzer N, et al. Management and Prognostic Markers in Patients With Autoimmune Encephalitis Requiring ICU Treatment. Neurol Neuroimmunol Neuroinflamm (2019) 6:e514. doi: 10.1212/NXI.0000000000000514
19. Lancaster E, Lai M, Peng X, Hughes E, Constantinescu R, Raizer J, et al Antibodies to the GABA(B) Receptor in Limbic Encephalitis With Seizures: Case Series and Characterisation of the Antigen. Lancet Neurol (2010) 9:6776. doi: 10.1016/S1474-4422(09)70324-2

20. Maureille A, Fenouil T, Joubert B, Picard G, Rogemond V, Pinto AL, et al. Isolated Seizures are a Common Early Feature of Paraneoplastic Anti-GABAB Receptor Encephalitis. J Neurol (2019) 266:195-206. doi: 10.1007/s00415-018-9132-0

21. van Sonderen A, Thijs RD, Coenders EC, Jiskoot LC, Sanchez E, de Bruijn MA, et al. Anti-LGI1 Encephalitis: Clinical Syndrome and Long-Term FollowUp. Neurology (2016) 87:1449-56. doi: 10.1212/WNL.0000000000003173

22. Gong X, Chen C, Liu X, Lin J, Li A, Guo K, et al. Long-Term Functional Outcomes and Relapse of Anti-NMDA Receptor Encephalitis: A Cohort Study in Western China. Neurol Neuroimmunol Neuroinflamm (2021) 8. doi: 10.1212/NXI.0000000000000958

23. Harutyunyan G, Hauer L, Dunser MW, Moser T, Pikija S, Leitinger M, et al. Risk Factors for Intensive Care Unit Admission in Patients With Autoimmune Encephalitis. Front Immunol (2017) 8:835. doi: 10.3389/fimmu.2017.00835

24. Thakur KT, Motta M, Asemota AO, Kirsch HL, Benavides DR, Schneider EB, et al. Predictors of Outcome in Acute Encephalitis. Neurology (2013) 81:793800. doi: 10.1212/WNL.0b013e3182a2cc6d

25. de Montmollin E, Demeret S, Brule N, Conrad M, Dailler F, Lerolle N, et al. Anti-N-Methyl-D-Aspartate Receptor Encephalitis in Adult Patients Requiring Intensive Care. Am J Respir Crit Care Med (2017) 195:491-9. doi: 10.1164/rccm.201603-0507OC

26. Wang W, Li JM, Hu FY, Wang R, Hong Z, He L, et al. Anti-NMDA Receptor Encephalitis: Clinical Characteristics, Predictors of Outcome and the Knowledge Gap in Southwest China. Eur J Neurol (2016) 23:621-9. doi: 10.1111/ene.12911

27. Zhang Y, Liu G, Jiang M, Chen W, He Y, Su Y. Clinical Characteristics and Prognosis of Severe Anti-N-Methyl-D-Aspartate Receptor Encephalitis Patients. Neurocrit Care (2018) 29:264-72. doi: 10.1007/s12028-018-0536-6

28. van de Riet EH, Esseveld MM, Cuypers L, Schieveld JN. Anti-NMDAR Encephalitis: A New, Severe and Challenging Enduring Entity. Eur Child Adolesc Psychiatry (2013) 22:319-23. doi: 10.1007/s00787-012-0351-0

29. Hoftberger R, Titulaer MJ, Sabater L, Dome B, Rozsas A, Hegedus B, et al. Encephalitis and GABAB Receptor Antibodies: Novel Findings in a New Case Series of 20 Patients. Neurology (2013) 81:1500-6. doi: 10.1212/ WNL.0b013e3182a9585f

30. Sun GW, Shook TL, Kay GL. Inappropriate Use of Bivariable Analysis to Screen Risk Factors for Use in Multivariable Analysis. J Clin Epidemiol (1996) 49:907-16. doi: 10.1016/0895-4356(96)00025-X

31. Sauerbrei W, Perperoglou A, Schmid M, Abrahamowicz M, Becher H, Binder $\mathrm{H}$, et al. State of the Art in Selection of Variables and Functional Forms in Multivariable Analysis-Outstanding Issues. Diagn Progn Res (2020) 4.3 doi: $10.1186 /$ s41512-020-00074-3

Conflict of Interest: The authors declare that the research was conducted in the absence of any commercial or financial relationships that could be construed as a potential conflict of interest.

Publisher's Note: All claims expressed in this article are solely those of the authors and do not necessarily represent those of their affiliated organizations, or those of the publisher, the editors and the reviewers. Any product that may be evaluated in this article, or claim that may be made by its manufacturer, is not guaranteed or endorsed by the publisher.

Copyright (c) 2022 Zhong, Chen, Zhang, Zhang and Lin. This is an open-access article distributed under the terms of the Creative Commons Attribution License (CC BY). The use, distribution or reproduction in other forums is permitted, provided the original author(s) and the copyright owner(s) are credited and that the original publication in this journal is cited, in accordance with accepted academic practice. No use, distribution or reproduction is permitted which does not comply with these terms. 